\title{
Hadronic tau decay at BaBar
}

\section{Olga Igonkina* for the BaBar collaboration}

University of Oregon, USA

E-mail: olya@slac.stanford.edu

Recent results on hadronic decays of tau from BaBar are reviewed. The branching fraction of $\tau^{-} \rightarrow 3 h^{-} 2 h^{+} v_{\tau}$ is measured to be $\left(8.56 \pm 0.05_{\text {stat }} \pm 0.42_{\text {sys }}\right) \times 10^{-4}$. The underlaying substructure of this decay exposes strong contribution from $\rho$ resonance. The decay $\tau^{-} \rightarrow f_{1}(1285) \pi^{-} v_{\tau}$ with $f_{1}(1285) \rightarrow 2 \pi^{-} 2 \pi^{+}$is observed and the corresponding branching fraction is measured to be $\left(3.9 \pm 0.7_{\text {stat }} \pm 0.5_{\text {sys }}\right) \times 10^{-4}$. The search for $\tau^{-} \rightarrow 4 \pi^{-} 3 \pi^{+}\left(\pi^{0}\right) \nu_{\tau}$ decay was performed and without evidence of the signal the upper limit of $B\left(\tau^{-} \rightarrow 4 \pi^{-} 3 \pi^{+}\left(\pi^{0}\right) \nu_{\tau}\right)$ is estimated to be $3.0 \times 10^{-7}$ at $90 \%$ confidence limit. The upper limits on branching fractions for the exclusive decays $\tau^{-} \rightarrow 4 \pi^{-} 3 \pi^{+} \nu_{\tau}$ and $\tau^{-} \rightarrow 4 \pi^{-} 3 \pi^{+} \pi^{0} \nu_{\tau}$ are found to be $4.3 \times 10^{-7}$ and $2.5 \times 10^{-7}$ at $90 \%$ confidence limit, respectively.

International Europhysics Conference on High Energy Physics

July 21 st - 27th 2005

Lisboa, Portugal

* Speaker. 


\section{Introduction}

Tau lepton decays provide very clean environment to test Standard Model, to study QCD and hadronization process as they probe the matrix element of the left-handed current between vacuum and the hadronic state. Many interesting results such as measurements of tau and tau neutrino masses, observation of low mass resonances, test of $\mathrm{CP}$ violation in lepton sector, searches for physics beyond Standard Model were obtained with tau decays into one and three charged hadrons in the final state (see e.g. Ref. [1]). In this paper we present a study of five prong tau decays which reveals underlying resonance structure and a search for seven prong tau decays done by BaBar experiment.

The data sample for the analyzes were collected with the BaBar detector at the PEP2 asymmetricenergy $e^{+} e^{-}$storage ring. The sample consists of $232 \mathrm{fb}^{-1}$ at luminosity-weighted center-of-mass $(C M)$ energy $\sqrt{s}=10.58 \mathrm{GeV}$. With an estimated cross section for tau pairs of $\sigma_{\tau \tau}=(0.89 \pm 0.02)$ $\mathrm{nb}$ [2], this data sample contains over 400 million tau decays. The BaBar detector is described in detail elsewhere [3].

\section{Measurement of $B\left(\tau^{-} \rightarrow 3 h^{-} 2 h^{+} v_{\tau}\right)$}

The analysis technique for study of 5-prong tau decays utilizes the fact that decays of two taus in the event at given $\sqrt{s}$ are well separated in space. One (signal) hemisphere, as defined by plane perpendicular to the event thrust axis in $\mathrm{CM}$, must contain five charged tracks while number of charged tracks in second (tag) hemisphere must be exactly one (1-5 topology). All tracks are required to point to the primary vertex and the net charge must be zero. The tag side track is required to satisfy electron or muon identification criteria and have a CM momentum less than $4 \mathrm{GeV} / c$ while the signal side tracks can not pass electron identification. No $\pi^{0}$ candidate is allowed on the signal side. The neutral energy deposited in the tag hemisphere is required to be less than $1 \mathrm{GeV}$. The magnitude of thrust, the total transverse momentum and the missing energy in the event are used to suppress backgrounds.

The selected sample consists of about 35000 events. The main sources of the background are 3-prong tau decays and multi-hadronic events. The background contamination is about $20 \%$ as estimated from Monte Carlo simulation $(M C)$. Table 1 shows number of events, background contribution, signal efficiency and the branching fraction for each lepton tag. The systematic errors include uncertainties on the background contribution, on the reconstruction efficiency, on the total number of tau decays and on the lepton identification. The average branching fraction is $(8.56 \pm$ $\left.0.05_{\text {stat }} \pm 0.42_{\text {sys }}\right) \times 10^{-4}$ which is in good agreement with the PDG value [4]

\begin{tabular}{lcc}
\hline & $e$-tag & $\mu$-tag \\
\hline$N_{\text {evt }}$ & 20920 & 13929 \\
$\varepsilon[\%]$ & $4.71 \pm 0.05$ & $3.03 \pm 0.04$ \\
$f_{\text {bgr }}[\%]$ & $20.6 \pm 2.0$ & $21.7 \pm 2.1$ \\
\hline$B\left(\tau^{-} \rightarrow 3 h^{-} 2 h^{+} v_{\tau}\right)\left[10^{-4}\right]$ & $8.53 \pm 0.06_{\text {stat }} \pm 0.42_{\text {sys }}$ & $8.73 \pm 0.07_{\text {stat }} \pm 0.48_{\text {sys }}$ \\
\hline
\end{tabular}

Table 1: The number of events, the background contribution, the signal efficiency and the branching fraction for electron and muon tagged samples for $\tau^{-} \rightarrow 3 h^{-} 2 h^{+} v_{\tau}$ analysis. 
The observed number of five prong tau decays is significantly larger than 295 events previously reported by CLEO [5] and allows for a first time a study of an underlying substructure. As one can see from Fig. 1a the reconstructed invariant mass of the five charged hadrons is not described by the phase space distribution as assumed in the MC. All tracks are taken as pions. Moreover, the invariant mass of two oppositely charged pions clearly exposes the contribution from the $\rho$ resonance (see Fig. 1b).

Further details of the analysis could be found in Ref. [6].
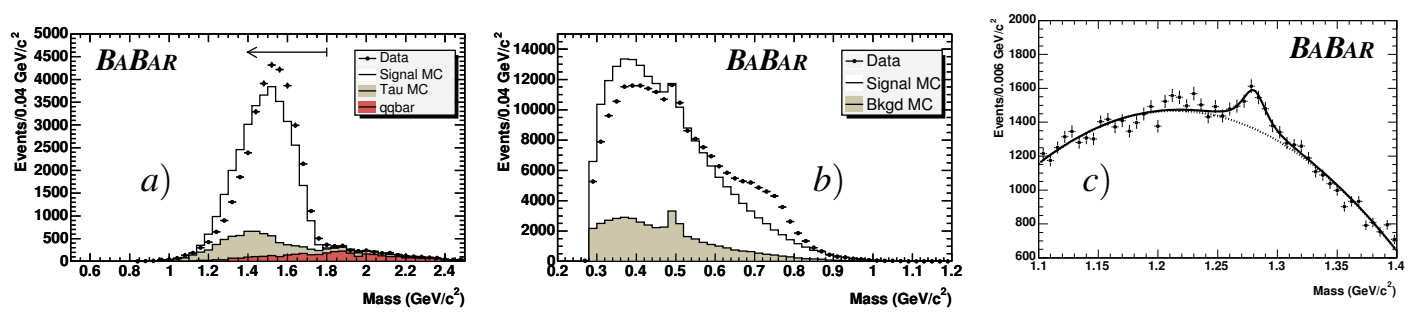

Figure 1: Invariant mass of $a$ ) five charged particles $b) h^{+} h^{-}$pairs $c$ ) $2 h^{+} 2 h^{-}$combinations. All tracks are taken as pions. The points are the data and the histograms are the Monte Carlo simulation. The unshaded and shaded histograms are the signal and background events, respectively. The Monte Carlo sample is normalized to the luminosity of the data sample. The solid line on plot $c$ is a fit to the data as described in the text.

\section{Measurement of $B\left(\tau^{-} \rightarrow f_{1}(1285) \pi^{-} \nu_{\tau}\right)$}

The sample described in sec. 2 is used also to measure branching fraction of $\tau^{-} \rightarrow f_{1}(1285) \pi^{-} v_{\tau}$, where $f_{1}(1285)$ consequently decays into four charged pions. Clear signal is observed (see Fig. 1c) and the distribution is fit with a second order polynomial for the background and a Breit-Wigner convoluted with a Gaussian for the signal. The width of the Gaussian is set to the expected mass resolution. The background distribution is determined from the fit with excluded peak region $\left(1.25-1.31 \mathrm{GeV} / c^{2}\right)$. A total of $1369 \pm 232$ signal events are obtained from the fit. The fraction of $\tau^{-} \rightarrow f_{1}(1285) \pi^{-} v_{\tau}$ decays found in $\tau^{-} \rightarrow 3 h^{-} 2 h^{+} v_{\tau}$ decays is measured to be $(0.050 \pm$ $\left.0.008_{\text {stat }} \pm 0.005_{\text {sys }}\right)$ and the branching fraction of $\tau^{-} \rightarrow f_{1}(1285) \pi^{-} v_{\tau}$ is calculated to be $(3.9 \pm$ $\left.0.7_{\text {stat }} \pm 0.5_{\text {sys }}\right) \times 10^{-4}$ where $B\left(f_{1}(1285) \rightarrow 2 \pi^{-} 2 \pi^{+}\right)$is taken from PDG [4]. The sources of systematic uncertainty are the uncertainties in the background shape and in the branching fractions of $\tau^{-} \rightarrow 3 h^{-} 2 h^{+} v_{\tau}$ and $f_{1}(1285) \rightarrow 2 \pi^{-} 2 \pi^{+}$decays.

\section{Search for $\tau^{-} \rightarrow 4 \pi^{-} 3 \pi^{+}\left(\pi^{0}\right) \nu_{\tau}$ decay}

The procedure to select seven prong tau decays is similar to one described in sec. 2 . In this case, 1-7 topology events are selected and the tag side must be compatible with an electron, muon or rho tau decay. The signal is sought in the distribution of pseudo mass defined as

$$
m^{* 2}=2\left(E_{\text {heam }}-E_{7 \pi}\right)\left(E_{7 \pi}-p_{7 \pi}\right)+m_{7 \pi}^{2},
$$


where $E_{\text {beam }}$ is an energy of the beam in $\mathrm{CM}$ and $\left(p_{7 \pi}, E_{7 \pi}\right)$ are $\mathrm{CM}$ momentum and energy of 7 charged pions in signal hemisphere. The signal is expected to peak in the region $1.3<m^{*}<$ $1.8 \mathrm{GeV} / \mathrm{c}^{2}$. The background from five prong tau decays is estimated with $\mathrm{MC}$ and the multihadron background is fit with Gaussian using the sideband of the pseudo mass data distribution $1.8<m^{*}<2.6 \mathrm{GeV} / c^{2}$.

24 events are observed in the signal region with $21.6 \pm 1.2$ events expected (see fig. 2. The signal efficiency is estimated to be $(9.4 \pm 0.6) \%$ using $\mathrm{MC}$ with the phase space distribution of tau decay products. In absence of the signal the branching fraction is found to be $B\left(\tau^{-} \rightarrow\right.$ $\left.4 \pi^{-} 3 \pi^{+}\left(\pi^{0}\right) v_{\tau}\right)<3.0 \times 10^{-7}$ at $90 \% \mathrm{CL}$. The obtained upper limit is ten times stricter than previous CLEO result [7].

The search was preformed also for exclusive decays $\tau^{-} \rightarrow 4 \pi^{-} 3 \pi^{+} \pi^{0} \nu_{\tau}$ and $\tau^{-} \rightarrow 4 \pi^{-} 3 \pi^{+} v_{\tau}$ by requiring or vetoing events with reconstructed $\pi^{0}$ or photon candidates. The corresponding signal efficiencies are $(3.6 \pm 0.3) \%$ and $(5.5 \pm 0.3) \%$. No signal is observed in either case. For $\tau^{-} \rightarrow 4 \pi^{-} 3 \pi^{+} \pi^{0} v_{\tau}$ sample we found seven events while expecting $8.2 \pm 0.5$ background events and for $\tau^{-} \rightarrow 4 \pi^{-} 3 \pi^{+} v_{\tau}$ eight events are observed while $3.9 \pm 0.8$ background events are expected (see fig. 2). The upper limits are set as

$$
\begin{aligned}
B\left(\tau^{-} \rightarrow 4 \pi^{-} 3 \pi^{+} \pi^{0} v_{\tau}\right) & <2.5 \times 10^{-7} 90 \% \mathrm{CL}, \\
B\left(\tau^{-} \rightarrow 4 \pi^{-} 3 \pi^{+} v_{\tau}\right) & <4.3 \times 10^{-7} 90 \% \mathrm{CL} .
\end{aligned}
$$

Further details of the analysis are given in Ref. [8].
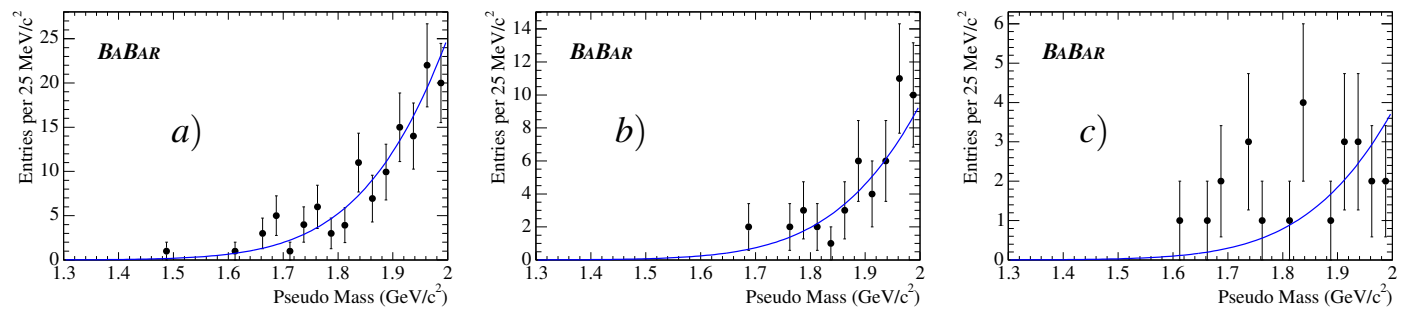

Figure 2: Pseudo mass spectra for a) $\tau^{-} \rightarrow 4 \pi^{-} 3 \pi^{+}\left(\pi^{0}\right) v_{\tau}$;b) $\left.\tau^{-} \rightarrow 4 \pi^{-} 3 \pi^{+} \pi^{0} v_{\tau} ; c\right) \tau^{-} \rightarrow 4 \pi^{-} 3 \pi^{+} v_{\tau}$. The points show data and the curve correspond to the fit as described in the text.

\section{References}

[1] A. Stahl, "Physics with tau leptons," Berlin, Germany: Springer (2000)

[2] B. F. Ward, S. Jadach, and Z. Was, Nucl. Phys. Proc. Suppl. 116, 73 (2003).

[3] B. Aubert et al. [BABAR Collaboration], Nucl. Instrum. Meth. A 479, 1 (2002)

[4] Particle Data Group, S. Eidelman et al., Phys. Lett. B592, 1 (2004).

[5] D. Gibaut et al. [CLEO Collaboration], Phys. Rev. Lett. 73, 934 (1994).

[6] B. Aubert et al. [BABAR Collaborations],

[7] K. W. Edwards et al. [CLEO Collaboration], Phys. Rev. D 56, 5297 (1997)

[8] B. Aubert et al. [BABAR Collaboration], Phys. Rev. D 72, 012003 (2005) 